\title{
1 Einführung: Zum Anliegen des Buches
}

Judenfeindschaft zeigt sich seit vielen Jahrhunderten nicht nur in physischer Gewalt und sozialer Diskriminierung, sondern auch in zahlreichen sprachlichen Äußerungen, die Juden stigmatisieren und diffamieren. Verbaler Antisemitismus manifestiert sich als Gewalt durch Sprache, Gewalt in Form von diskriminierenden, beleidigenden und realitätsverzerrenden Sprachhandlungen gegenüber Juden. Mittels Sprache werden Feindbilder konstruiert bzw. aufrechterhalten, spezifische Negativbilder von Juden vermittelt, uralte tradierte Stereotype weitergegeben. Die Sprache spielt bei der Aufrechterhaltung und der Vermittlung antisemitischer Ressentiments eine besondere Rolle: Sprachliche Äußerungen aktivieren bzw. konstruieren spezifische Konzeptualisierungen von Juden als fremde, seltsame und schlechte Wesen und evozieren negative Gefühle. Historisch tradiertes Wissen wird in kollektiv verankerten Konzepten mittels sprachlicher Formen erhalten, und über den Sprachgebrauch werden judeophobe Vorstellungen zum Teil über viele Jahrhunderte hinweg transportiert. Juden werden als Unmenschen, Teufel und Unholde bezeichnet und somit dämonisiert; sie werden als geldgierige Wucherer, hinterhältige Verschwörer und rachsüchtige Nutznießer charakterisiert und dadurch moralisch diskreditiert; sie werden fremd, arrogant, halsstarrig sowie anders genannt und auf diese Weise als Minderheit negativ diskriminiert; das Judentum wird assoziiert mit Attributen wie atavistisch, brutal, seltsam, den wahren Glauben negierend und damit als Religion delegitimiert. Wiederholte Diskurserfahrungen mit klischeebelasteten Äußerungen und Floskeln führen zur Konstruktion teils bewusster, teils unbewusster mentaler Repräsentationen, die sich zu permanenten Einstellungen, Glaubenssystemen und sogar kompletten Weltbildern verdichten können. Vielfach fehlt jedoch das Bewusstsein für diese über Generationen hinweg bestehenden Sprachgebrauchsmuster und ihr Wirkungspotenzial hinsichtlich Ausgrenzung und Entwertung. Dabei sind negative Einstellungen und Feindbildkonstruktionen gegenüber Juden (weder in der Vergangenheit noch in der Gegenwart) keineswegs nur an den Rändern der Gesellschaft zu finden, sondern auch in der (politisch nicht radikalen, ökonomisch gut situierten und gebildeten) Mitte. ${ }^{1}$ Oft wird in diesem Zusammenhang missachtet oder verkannt, wie gefährlich die Verwendung sprachlicher Mittel und Strukturen sein kann, zumal wenn diese alte Muster der Judenfeindschaft zum Ausdruck bringen und unreflektiert reproduziert werden.

1 In der Antisemitismusforschung beobachtet man schon seit Jahren, dass sich „die Zone der Akzeptanz von antisemitischen Vorurteilen in den letzten Jahren gewandelt oder vergrößert hat“ (Rensmann/Schoeps 2008: 19). S. hierzu auch Schwarz-Friesel et al. (2010) und SchwarzFriesel (2010a). Vgl. hierzu auch BMI (2011: 68). 
Es ist daher ein wichtiges Anliegen dieses Buches, die Macht und die Gewalt aufzuzeigen, die von Sprache bzw. ihrer spezifischen Verwendung ausgehen kann. Wir werden anhand umfangreicher empirischer Quellen zeigen, dass sich im aktuellen Sprachgebrauch trotz aller Aufklärungsarbeit nach dem Holocaust die gesamte Palette tradierter judenfeindlicher Stereotype und emotionaler Ressentiments abzeichnet und dass antisemitisches Gedankengut nicht nur von Rechts- und Linksextremisten artikuliert wird. Auch gebildete Menschen produzieren teils intentional, teils nicht-intentional Äußerungen mit judenfeindlichen Inhalten. Dabei sind unterschiedliche Typen von Verbal-Antisemitismen zu unterscheiden: Kommunikativ kann Judeophobie explizit, also manifest und offensichtlich, oder implizit, also indirekt und über Schlussfolgerungen und Kontextwissen erkennbar, ausgedrückt werden. Die spezifischen Charakteristika der aktuellen judenfeindlichen Sprachverwendung ${ }^{2}$ sind bislang nur unzureichend analysiert und beschrieben worden. ${ }^{3}$ Insbesondere die (zunehmend im öffentlichen Diskurs zu hörenden/lesenden) impliziten Formen des Verbal-Antisemitismus, die über Implikaturen kontextuell erschließbar sind, gehören zu den noch nicht hinreichend bekannten und erforschten Phänomenen: Das Wort Jude(n) muss keineswegs in einer Äußerung vorkommen, um diese als antisemitisch klassifizieren zu können. Es gibt viele verschiedene Möglichkeiten, über Anspielungen, bestimmte Paraphrasen oder die Verknüpfung spezifischer Argumente judenfeindliche Inhalte auszudrücken.

Das Buch behandelt die verschiedenen Verbalmanifestationen der aktuellen Judenfeindschaft und die ihnen zugrundeliegenden geistigen Vorstellungen zu Juden/Judentum sowohl aus (diskurs)historischer als auch aus sprach- und kognitionswissenschaftlicher Perspektive.

Eine Analyse der lexikalischen, semantischen, syntaktischen und argumentativ-konzeptuellen Besonderheiten des antisemitischen Sprachgebrauchs ist also nicht nur eine notwendige Voraussetzung für ein besseres Verständnis der in ihm zum Ausdruck kommenden Stereotype und emotional geprägten Vorurteile, sondern auch allgemein für ein umfassendes Verständnis des kulturell, sozial, kognitiv und emotional geprägten Phänomens der Judenfeindschaft. ${ }^{4}$

2 Die letzten detaillierten linguistischen Untersuchungen zum verbalen Nachkriegsantisemitismus auf empirischer Basis liegen zwanzig Jahre zurück (s. Wodak 1990 und Wodak et al. 1990, die Analysen zum Sprachgebrauch in Österreich vorgenommen hatten).

3 So stellt auch die Expertenkommission des Bundestages fest (BMI 2011: 72): „Während vergleichbare Phänomene antisemitischer Stereotypenbildung und der Verankerung antisemitischer Codes für vergangene Epochen relativ gut untersucht sind, besteht für die Alltagskultur der jüngsten Zeit, gerade unter dem Gesichtspunkt des 'modernisierten Antisemitismus', noch erheblicher Forschungsbedarf.“

4 Vgl. BMI (2011: 179): „Angesichts der relativ weiten Verbreitung antisemitischer 
Angesichts der immer wieder aufflammenden, zum Teil heftig und stark emotional geführten Debatten in der deutschen Gesellschaft, ob eine mündliche oder schriftliche Äußerung überhaupt als antisemitisch einzustufen sei, ist es zudem auch gesellschaftlich von großer Relevanz, Klarheit zu schaffen und eindeutige Klassifikationskriterien für die Beantwortung dieser Frage vorzulegen. Neben der Beschreibung der Charakteristika der aktuellen Judenfeindschaft als Verbal-Antisemitismus ist es daher auch ein Ziel des Buches, textwissenschaftliche Klassifikationsindikatoren hinsichtlich der Frage ${ }^{5}$ „Wann ist eine sprachliche Äußerung antisemitisch?“ vorzulegen.

Empirische Basis unserer Untersuchung ist eine umfangreiche Korpusanalyse, die vor allem qualitativ ${ }^{6}$ ausgerichtet ist, wenngleich auch einige quantitative Erhebungen Berücksichtigung finden, sofern sie helfen, das Phänomen der modernen Manifestationen des verbalen Antisemitismus besser zu veranschaulichen. Der Studie liegt die Analyse von über 14.000 E-Mails und Briefen an den Zentralrat der Juden (aus den Jahren 2002 bis 2009) und an die Israelische Botschaft in Deutschland (aus den Jahren 2004 bis 2012) zugrunde, die Einblick in die Einstellungen, Gedanken und Gefühle der Schreiber geben und damit die modernen Formen der Judenfeindschaft ${ }^{7}$ im 21. Jahrhundert transparent machen. Ergänzt wird diese Korpusanalyse der E-Mails, Faxe und Briefe durch die Untersu-

Einstellungen in der Bevölkerung und der Präsenz von Judenfeindschaft im Alltag stellt sich [...] die bisher vollkommen unbeantwortete Frage, wie antisemitische Stereotype, Vorurteile und Einstellungen in unserer Gesellschaft tradiert werden. Von der Beantwortung dieser Frage hängt insbesondere die Entwicklung wirksamer Gegenstrategien ab.“

5 Angesichts der immer wieder aufs Neue geführten, die Wissenschaft zumeist völlig ignorierenden Debatten (s. zuletzt die intensive Debatte um den israel-feindlichen Text von Günter Grass) sowie der zahlreichen Beleidigungsklagen und Gerichtsprozesse, ist es von nicht unerheblicher Relevanz, wenn die Wissenschaft klar Auskunft darüber geben kann, wann eine Äußerung als antisemitisch einzustufen ist. Zurzeit wird bei nahezu jedem „sprachlichen Delikt“" nach einer Ausrede oder Umdeutung gesucht, gerieren sich die Sprachproduzenten oft als Opfer einer angeblichen „Antisemitismus-Keule“. Eine gesellschaftlich auf breiter Basis bekannte, wissenschaftliche Klassifikation von Verbal-Antisemitismus könnte hier also hilfreich sein.

6 Korpusanalysen sind mittlerweile in den Sprach- und Kognitionswissenschaften die wichtigste Methodik zur systematischen und repräsentativen Erfassung sprachlicher und kognitiver Phänomene. Eine qualitative Korpusanalyse konzentriert sich auf die Ermittlung, Klassifizierung und Interpretation sprachlicher Phänomene. Eine quantitative Analyse hingegen richtet sich auf die Eruierung von Frequenzen bestimmter Phänomene (vgl. hierzu auch Scherer 2006: 36 f., Bubenhofer 2009). Nur qualitative Korpusanalysen können also die inhaltlichen Grundlagen aktueller Judenfeindschaft erfassen.

7 Die Mehrzahl der Schreiben ist als judenfeindlich einzustufen, wenngleich es auch eine Reihe von Zuschriften gibt, die entweder philosemitisch oder einfach solidarisch-unterstützend sind (s. hierzu die Angaben in Kap. 2.2). 
chung repräsentativer Sprachgebrauchsbeispiele aus dem öffentlichen, massenmedialen Diskurs (z. B. anhand der Textsorten Leserbriefe, Zeitungsartikel, Plakattexte, Blogeinträge). Diese stichprobenartig erhobenen Daten belegen, dass die Stereotype und Argumentationsmuster keineswegs auf die Textsorte E-Mail/ Brief beschränkt, sondern typisch für weite Teile der öffentlichen Kommunikation sind. Somit können wir anhand einer repräsentativen Studie ${ }^{8}$ zeigen, wie sich Judenfeindschaft im aktuellen Sprachgebrauch manifestiert, und erörtern, inwiefern sich die Interaktion von kognitiver Kategorisierung und emotionaler Bewertung auf den verschiedenen Strukturebenen dieser Muster zeigt. Damit erfassen wir nicht nur die verbalen Charakteristika des antisemitischen Sprachgebrauchs, sondern auch die zugrundeliegenden konzeptuellen Einstellungen. Sie transparent zu machen, ist besonders wichtig, da nur über ihre Erfassung erklärt werden kann, was Judenfeindschaft als mentales und affektives Phänomen auszeichnet. Die moderne Antisemitismusforschung muss zukünftig diesem Anspruch stärker gerecht werden und vermehrt qualitative Diskursanalysen durchführen.

Die Fragen, die sich vorrangig bei der textwissenschaftlichen Analyse der Korpusdaten stellen, beziehen sich auf die verbalen Charakteristika des antisemitischen Sprachgebrauchs sowie die ihnen zugrundeliegenden geistigen Kategorisierungs- und Bewertungsstrukturen: Welche (tradierten) anti-jüdischen Stereotype spiegeln sich in den aktuellen Texten explizit und/oder implizit wider und in welchen (neuen) Konzeptkombinationen treten sie auf? Welche emotionalen Einstellungen liegen der Verbalisierung judenfeindlicher Gesinnungen zugrunde? Mittels welcher argumentativen Strategien werden antisemitische Inhalte artikuliert und gerechtfertigt? Welche Mittel und Strukturen in Texten sind es, die judenfeindliche Inhalte darstellen und ausdrücken? Welche dieser Ausdrucksformen vermitteln per se, also allein über die Semantik, kontextfrei Antisemitismus, welche dagegen ergeben erst im Kontext und kulturanalytisch betrachtet eine judenfeindliche Lesart?

Bei allen Analysen gehen wir von der Grundannahme aus, dass sprachliche Äußerungen Spuren der kognitiven und emotionalen Aktivität derjenigen sind, die sie produziert haben. Die Texte geben entsprechend Aufschluss über die Denkstrukturen, Einstellungen und Gefühle ihrer Verfasser. Dem Buch liegt insgesamt ein interdisziplinärer Untersuchungsansatz zugrunde: Wir verbinden geschichtswissenschaftliche Reflexion und sprachwissenschaftliche Text-

8 Das Kriterium der Repräsentativität bezieht sich hier auf das themenspezifische Korpus und damit auf den antisemitischen Sprachgebrauch. Die Korpusdaten sind repräsentativ für genau diesen Diskurs. Sie zeigen, welche Mittel und Strategien typisch für judenfeindliche Sprache in Deutschland sind. Das bedeutet nicht, dass sie repräsentativ für die gesamte Kommunikation über Juden in Deutschland wären oder etwa Angaben über alle Deutschen machen würden. 
analyse, um das Phänomen der Judenfeindschaft eingehend, in seinem historischen Kontext so präzise wie möglich in all seinen Ausprägungsvarianten zu erfassen. Bislang liegt in der Antisemitismusforschung ein solcher Ansatz noch nicht vor, wenngleich das Desiderat dafür bereits öfter genannt wurde. Nur historisch, soziologisch oder allgemein philologisch orientierte Abhandlungen zur Judenfeindschaft lassen zumeist textuelle Detailanalysen vermissen. Viele wichtige Aspekte werden zudem nicht präzise genug erklärt. Es finden sich oft lediglich sehr allgemeine und vage Aussagen zu antisemitischen Äußerungen, und viele Phänomene werden nicht hinreichend erklärt, zumal häufig Begriffe wie „Chiffre“, „Klischee“, „Stereotyp“ (oft gar wie Synonyme) benutzt werden, ohne dass sie hinreichend definiert und voneinander abgegrenzt werden. Auch Begriffe wie „latent“, „manifest“, „implizit“, „explizit“, „direkt“, „indirekt“ etc. werden außerhalb der Linguistik zumeist heterogen benutzt und bilden dann die Basis für Verwirrung und Unklarheit. Andererseits ergehen sich rein linguistisch ausgerichtete Abhandlungen zu Verbal-Antisemitismus oft nur in deskriptiven Aufzählungen von stilistisch-rhetorischen, lexikalischen und syntaktischen Besonderheiten, ohne diese in ihrer Kontextabhängigkeit, ihrer langjährigen kulturellen Einbettung und kognitiven Verankerung sowie ihrer kommunikativmanipulativen Relevanz zu erklären. Zudem erschwert oft der sehr spezifische fachwissenschaftliche Jargon die Verständlichkeit von sprachwissenschaftlichen Detailanalysen und macht sie daher in der Rezeption wenig attraktiv bzw. schwer zugänglich für Nicht-Linguisten. Wir möchten in diesem Buch einerseits die für wissenschaftliche Textanalysen sprachlicher Äußerungen notwendige Präzision mittels klarer Erläuterungen und exakter Verwendung von Fachtermini einhalten, andererseits aber diese Erläuterungen insgesamt so verständlich wie möglich formulieren. Alle theoretisch und wissenschaftlich relevanten Aspekte werden stets anhand von konkreten Beispielen aus dem Korpus erörtert. Wir sind davon überzeugt, dass gerade die vielen authentischen Äußerungsbeispiele dem Leser einen besonders anschaulichen Eindruck des Potenzials judenfeindlicher Sprache vermitteln können. Die übergeordneten Reflexionen aus geschichts- und kognitionswissenschaftlicher Perspektive sollen dabei helfen, das Phänomen der modernen Judenfeindschaft im Rahmen seiner soziokulturellen Verankerung und seiner kognitiv-emotionalen Interaktion zu verstehen.

Wir werden, nach einer kurzen Abhandlung zur Relevanz und Funktion von Sprache bei der Etablierung sowie Tradierung judenfeindlicher Inhalte, zunächst anhand von historischen Texten aufzeigen, inwiefern bestimmte mentale Stereotype über alle Epochen hinweg im kulturellen Gedächtnis sowie der kommunikativen Praxis erhalten und sprachlich auf eine erstaunlich homogene Weise wiederholt werden. Wir gehen dabei auch auf die Genese von Judenhass ein. Nach einer Charakterisierung judenfeindlicher Äußerungsformen aus dem 19. und 20. 
Jahrhundert, deren Kenntnis für ein Verständnis des Antisemitismus als einem kontinuierlich auftretenden Phänomen unerlässlich ist, konzentrieren wir uns auf aktuelle verbale Realisierungen antisemitischen Gedankengutes in den ersten zehn Jahren des 21. Jahrhunderts in Deutschland. Hierfür ist zu konstatieren, dass der Holocaust keineswegs die grundlegende Zäsur in Bezug auf die Überwindung von Vorurteilsstrukturen und Hassgefühlen gegenüber Juden darstellte, wie weithin angenommen wird, da eine tiefgreifende, selbstkritische Reflexion und eine alle Gesellschaftsstrukturen umfassende Vergangenheitsaufarbeitung nach 1945 nicht so stattfand, wie der Zivilisationsbruch es erfordert hätte.

Entsprechend treten die meisten konzeptuellen Muster der zweitausend Jahre alten Judenfeindschaft ungebrochen teils in überlieferten, teils in neuen Ausdrucksvarianten auf. Wir werden in diesem Zusammenhang auch zeigen, dass bis heute viele Versatzstücke (Wörter, Phrasen, Metaphern) aus dem ideologiegeprägten NS-Sprachgebrauch reproduziert werden. Die modernen judenfeindlichen Stereotype, Argumente und Strategien sowie deren sprachliche Realisierungen werden in ihrem Kontext beschrieben und erklärt. Erörtert wird dabei auch, inwiefern der Anti-Israelismus die dominante Formvariante des aktuellen VerbalAntisemitismus darstellt und wie über die Bezugnahme auf den jüdischen Staat Israel generell Judenhass artikuliert wird. Eine kurze Vergleichsstudie zu verbalantisemitischen Texten anderer europäischer Länder schließt sich an und lenkt den Blick auf die wesentlichen konzeptuellen Gemeinsamkeiten judenfeindlicher Gesinnungen in Europa. Im deutschen Diskurs wird Judenfeindschaft aufgrund der NS-Vergangenheit allerdings wesentlich stärker durch emotionale Schuld-, Scham- und Erinnerungsabwehrtendenzen, Verantwortungsverdrängung und eine Überdruss-Mentalität bestimmt. Daher werden wir auch auf die emotionale Dimension des modernen Judenhasses eingehen und zeigen, welche Gefühle (in Interaktion mit stereotypen Denkstrukturen) die affektive und irrationale Grundlage von Judenfeindschaft bilden. Judenfeindschaft basiert maßgeblich auf einem tief sitzenden, von der abendländischen Kultur geprägten Ressentiment, das sich als sehr resistent gegenüber Fakten, Aufklärung und Argumentation erweist. Umso wichtiger ist es, die diversen Formen von Verdrängung, Leugnung und Umdeutung im modernen antisemitischen Diskurs nicht nur zu beschreiben, sondern auch in der Breite der Gesellschaft das kritische Bewusstsein für ihre Existenz sowie Frequenz zu schaffen und damit Möglichkeiten ihrer Bekämpfung zu entwickeln. 